\title{
Novel Measure of the Weigh Distribution Balance on the Brain Network: Graph Complexity Applied to Schizophrenia
}

\author{
J. Gomez-Pilar, Student Member, IEEE, A. Bachiller, P. Nuñez-Novo, J. Poza, Senior Member, IEEE, \\ C. Gómez, Senior Member, IEEE, A. Lubeiro, V. Molina, R. Hornero Senior Member, IEEE
}

\begin{abstract}
The aim of this study was to assess brain complexity dynamics in schizophrenia $(\mathrm{SCH})$ patients during an auditory oddball task. For that task, we applied a novel graph measure based on the balance of the node weighs distribution. Previous studies applied complexity parameters that were strongly dependent on network topology. This fact could bias the results besides being necessary correction techniques as surrogating process. In the present study, we applied a novel graph complexity measure from the information theory: Shannon Graph Complexity (SGC). Complexity patterns form electroencephalographic recordings of 20 healthy controls and $20 \mathrm{SCH}$ patients during an auditory oddball task were analyzed. Results showed a significantly more pronounced decrease of SGC for controls than for SCH patients during the cognitive task. These findings suggest an important change in the brain configuration towards more balanced networks, mainly in the connections related to longrange interactions. Since these changes are significantly more pronounced in controls, it implies a deficit in the neural network reorganization in $\mathrm{SCH}$ patients. In addition, SGC showed a suitable discrimination ability using a leave-one-out cross-validation: 0.725 accuracy and 0.752 area under receiver operating characteristics curve. The novel complexity measure proposed in this study demonstrated to be independent of network topology and, therefore, it complements traditional graph measures to characterize brain networks.
\end{abstract}

\section{INTRODUCTION}

Higher-order mental functions, such as cognitive tasks, depend of the global coordination of cerebral activity. In this regard, several studies of schizophrenia $(\mathrm{SCH})$ are based on the hypothesis that this pathology produces a disruption on the synaptic efficacy [1], which results on functional disconnection during working memory processing [2]. Although, previous studies suggest that $\mathrm{SCH}$ is accompanied by a reorganization deficit of the brain network during the performance of a simple cognitive task, such as an auditory

\footnotetext{
**This research was supported in part by the projects TEC2014-53196-R of 'Ministerio de Economía y Competitividad' and FEDER, and the project VA059U13 of "Consejería de Educación". J. Gomez-Pilar and A. Bachiller were in receipt of a PIF-UVA grant from University of Valladolid and an additional scholarship from the University of Valladolid for attending conferences.

J. Gomez-Pilar, is with the Biomedical Engineering Group, University of Valladolid, Spain, (phone: 0034 983423000-5589; e-mail: javier.gomez@ gib.tel.uva.es).

A. Bachiller, P. Nuñez-Novo, J. Poza, C. Gómez and R. Hornero are with the Biomedical Engineering Group, University of Valladolid, Spain (emails: alejandro.bachiller@uva.es,_pablo.nunez@gib.tel.uva.es, jesus.poza@tel.uva.es, carlos.gomez@tel.uva.es, ).

V. Molina and A. Lubeiro are with the Faculty of Medicine, University of Valladolid, Avenida Ramón y Cajal 7, 47007 Valladolid, Spain (e-mail: vmolina@med.uva.es, alba.lubeiro@gmail.com).
}

oddball task [3], new research is required to clearly demonstrate this statement.

In this context, new methods for assessing brain networks have been introduced. Most of the studies of brain networks used magnetic resonance imaging (MRI) to achieve a suitable spatial resolution [4]. Nevertheless, neural mechanisms underlying cognitive processing are related to fast changes in the spatio-temporal patterns [5]. The temporal resolution provided by electroencephalogram (EEG) can then complement previous MRI analyses, assessing brain dynamics. Although EEG recordings can be biased by the volume conduction effect among spatially contiguous sensors, the comparison of different conditions (e.g. during different times of a cognitive task) and the use of highdensity EEG recordings may help to overcome this issue.

Complex Network Theory is a useful tool to assess global and local brain connectivity, which seems to be altered in $\mathrm{SCH}$ [6]. Several studies were devoted to characterizing brain networks dynamics. Some of them focused on the complexity of a graph $[7,8]$. Nonetheless, the underlying issue is how to define 'graph complexity'. Several studies suggest that a network with high complexity must stand halfway between segregation and integration [7], whereas others propose that it must be related to the small-world concept (halfway between lattice and random graphs) [8]. All these definitions are very influenced by network topology, so the complementary to traditional graph measures remains in the background. In the current research, we used a definition of complexity that captures regularities based on the deviation from a totally balanced weigh distribution and totally unbalanced. Thereby, it does not depend on network topology.

Given the fact that the brain network connectivity is strongly dependent on the weigh distribution of the connections, we hypothesized that a complexity measure based on the weigh balance distribution among nodes would show an abnormal neural network reorganization during a cognitive task in $\mathrm{SCH}$.

\section{MATERIALS AND METHODS}

\section{A. EEG recordings}

Twenty SCH patients and 20 healthy controls took part in the study. Healthy controls (age- and gender-matched) were recruited through newspaper advertisements. SCH patients were diagnosed according to the Diagnostic and Statistical Manual of Mental Disorders, 5th edition [9] (DSM-V) criteria. The clinical status of the patients was scored using the Positive and Negative Syndrome Scale (PANSS) [10]. The study protocol was approved by the local Ethics Committee of Hospital Clínico Universitario from Valladolid 
(Spain) according to the code of ethics of the World Medical Association (Declaration of Helsinki).

Data acquisition was performed using an EEG system (BrainVision, Brain Products GmbH; Munich, Germany). Electrode placement followed the 10/20 system, with 32 electrodes at standard positions. Impedances were kept below $5 \mathrm{k} \Omega$. Event-related potential (ERP) recordings were performed while the participants were sat and with their eyes closed. The auditory oddball task consisted in random series of 600 tones with three different kinds of tones: target (500 $\mathrm{Hz}$ tone), distractor $(1000 \mathrm{~Hz}$ tone $)$ and standard $(2000 \mathrm{~Hz}$ tone) with probabilities of $0.20,0.20$ and 0.60 , respectively. Only target tones were considered, since they correspond to P3a waves.

ERP signals were recorded at a sampling frequency of $500 \mathrm{~Hz}$ during $13 \mathrm{~min}$ of auditory oddball task. Signals from TP9 and TP10 electrodes were removed because of muscle artifacts. An independent component analysis (ICA) was carried out using the remaining electrodes. According to a visual inspection of the scalp maps and their temporal activations, ICA components related to eyeblinks were discarded. Then, data were reconstructed and re-referenced over $\mathrm{Cz}$ electrode to the average activity of all active sensors in order to minimize the effect of microsaccadic artifacts [11]. Signals were filtered using a band-pass finite impulse response filter between 1 and $70 \mathrm{~Hz}$, as well as a $50 \mathrm{~Hz}$ notch filter. Finally, a two-steps artifact rejection algorithm was applied to minimize oculographic and myographic artifacts: (i) segmentation into $1 \mathrm{~s}$-length trials ranging from $-300 \mathrm{~ms}$ to $700 \mathrm{~ms}$ after stimulus onset; and (ii) automatic and adaptive trial rejection using a statistical-based thresholding method.

\section{B. Continuous Wavelet Transform}

ERP recordings are nonstationary signals that should be analyzed using a time-frequency representation [12]. Continuous Wavelet Transform (CWT) provides an alternative to classical Fourier analyses. In the present study, the complex Morlet wavelet was chosen as "mother wavelet", since it provides a biologically plausible fit to the ERP signals [3]. Thus, the Wavelet Scalogram (WS) was computed from the CWT of each trial [13]. WS summarizes the distribution of the energy for each trial. In order to interpret WS as a probability density function, it was normalized to range from 0 to 1 [13].

\section{Mean Squared Coherence}

The Mean Squared Coherence (MSC) can be used as a measure of the functional connectivity between brain regions [14]. In this study, MSC across electrodes was computed using the WS. Finally, MSC values were averaged in the conventional EEG frequency band: theta (4-8 Hz), alpha (813), beta-1 (13-19 Hz), beta-2 (19-30 Hz) and gamma (30-70 $\mathrm{Hz}$ ). Of note, delta band was not analyzed, since its associated wavelet duration is higher than the duration of the 1 s-length evoked response [13].

The MSC was computed from -300 to $700 \mathrm{~ms}$ from stimulus onset to avoid overlapping between trials. Then, the MSC was averaged in two windows: (i) the baseline window ([-300 0] $\mathrm{ms}$ from stimulus onset) and (ii) the response window ([150 450] ms after stimulus onset). The latter is related to P3a waves.

\section{Graph Complexity}

A functional network can be generated using MSC values as the weights of the links. Accordingly, the graph nodes can be associated to each electrode. In the current research, the network is composed by $N=30$ nodes, which are linked by edges with weights $w_{i j}$ ( $i$ and $j$ denote two different electrodes).

Several attempts have been previously conducted to quantitatively evaluate the graph complexity, some of them in the context of the information theory $[7,15]$. In the present work, we applied a novel complexity measure to characterize a graph: Shannon graph complexity $(S G C)$. To compute $\mathrm{SGC}$, it is firstly required to define the graph entropy, $H$, as a measure of the stochastic graph weight distribution. $H$ is given by the following formula [15]:

$$
H=\frac{-1}{\log _{2}\left(\begin{array}{c}
N \\
2
\end{array}\right)} \sum_{i=1}^{N} \sum_{\substack{j=1 \\
i \neq j}}^{N} \frac{w_{i j}}{W} \log _{2} \frac{w_{i j}}{W},
$$

where $W$ represents the sum of all weights of the graph and $\log _{2}\left(\begin{array}{l}N \\ 2\end{array}\right)$ is a normalization factor to ensure that $0 \leq H \leq 1 . H$ does not depend on the network size, enabling comparisons between studies with different number of nodes. Secondly, Shannon graph disequilibrium $(D)$ is defined as the statistic distance, in the probability space, between the equilibrium distribution and the distribution of the graph under study [16]. It is noteworthy that the uniform distribution is considered as the equilibrium distribution in Gibbs' statistical mechanics [16]. Thereby, a highly balanced weighted graph (as a graph with all weights with the same value) yields maximum Shannon graph entropy, $H^{\text {balanced }}=1$, and a low $D$ value. On the contrary, a highly unbalanced weighted graph yields $H^{\text {unbalanced }} \approx 0$ and a high $D$ value. In this study, Euclidean distance as used to compute $D$ as follows:

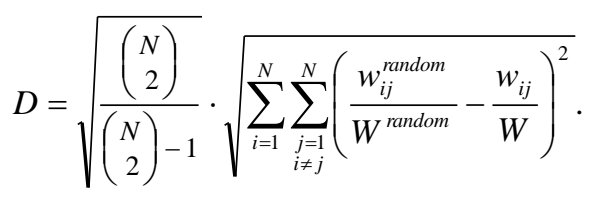

$D$ was normalized as in equation (1) to take values in the $0-1$ interval by dividing by its maximum value [16].

Finally, $S G C$ was calculated to capture the interplay between $H$ and $D$. This complexity measure considers that a graph weight distribution has two extreme states: totally balanced and totally unbalanced. Therefore, $S G C$ must be close to zero for those two extremes. $S G C$ is then defined as follows:

$$
S G C=H \cdot D .
$$

This novel measure simultaneously quantifies the distance between the considered graph to both a totally balanced and totally unbalanced weighted graph. Classical network measures, as characteristic path length $(C P L)$ or clustering coefficient $(C L C)$, are strongly influenced by topological features [17]. However, SGC are totally independent of 
network topology. This idea can be appreciated in Fig. 1 that depicts several graphs with their corresponding values of $H$, $D, S G C, C P L$ and $C L C$. Of note, maxima SGC values are obtained by whether the weight distribution is not totally balanced or unbalanced (central panels).

\section{B. Statistical Analysis}

Initially, an exploratory analysis was carried out to analyze data distribution. The normality and the homoscedasticity of the network parameter distributions were tested using the Kolmogorov-Smirnov test and the Levene test, respectively. We found that parametric test assumptions were not met. Nonparametric test were used to assess statistical differences: (i) Wilcoxon signed-rank test was used to compare baseline and response $S C G$ values for withingroup analyses; and (ii) Mann-Whitney $U$-test was used for between-group analyses. Finally, the SGC discrimination ability was assessed by means of a Receiver Operating Characteristic (ROC) curve using leave-one-out crossvalidation (LOO-CV).

\section{RESULTS AND DISCUSSION}

The $S G C$ was computed for the five conventional EEG frequency bands in the two windows under study: baseline and response. Then, the $S G C$ values in the baseline window were subtracted from the SGC values in the response window in order to compute the complexity change due to the cognitive oddball task. Only results on theta frequency band showed significant differences $(p<0.05)$. On one hand, Fig. 2.a shows the distribution of $S G C$ values for each window group in the theta band. A significant decrease on $S G C$ ( $p=$ $7.78 \cdot 10^{-4}$, Wilcoxon signed-rank test) was obtained in the control group from baseline to response window. Although, $\mathrm{SCH}$ group also shows a decrease in $S G C$ values, nonsignificant differences were found $(p>0.05$, Wilcoxon signed-rank test). Similarly, nonsignificant differences were found either in the comparisons between baseline windows for both groups or the comparisons between response windows for both groups ( $p>0.05$, Mann-Whitney $U$-test). On the other hand, Fig. 2.b shows the dynamical changes in complexity for both groups. Significant differences were obtained between the changes on $S G C$ for controls and patients $\left(p=2.49 \cdot 10^{-2}\right.$, Mann-Whitney $U$-test). These results suggest that an important reconfiguration on brain network during the cognitive task can be seen for controls. However, this change seems to be significantly lower for $\mathrm{SCH}$ group.

In regard with the physiological implications of the previous results, our findings seem to indicate three different aspects. Firstly, an important deficit on neural reorganization can be observed for SCH patients. This result is in line with the disconnection hypothesis of $\mathrm{SCH}$, which claims that the abnormal underlying substrates in $\mathrm{SCH}$ cause the dysfunctional integration among neuronal systems [18]. Secondly, our results suggest that the network reorganization elicited by the cognitive task can be associated with a decrease in network complexity. As previously mentioned, $S G C$ computes the distance between brain network under study and totally balanced and unbalanced networks. In addition, $H$ indicates the direction of $S G C$. For the control group, $H$ achieved values of $0.937 \pm 0.007$ during baseline window and $0.945 \pm 0.006$ during the active response. For $\mathrm{SCH}$ group, $H$ also showed an increase of weigh regularity:

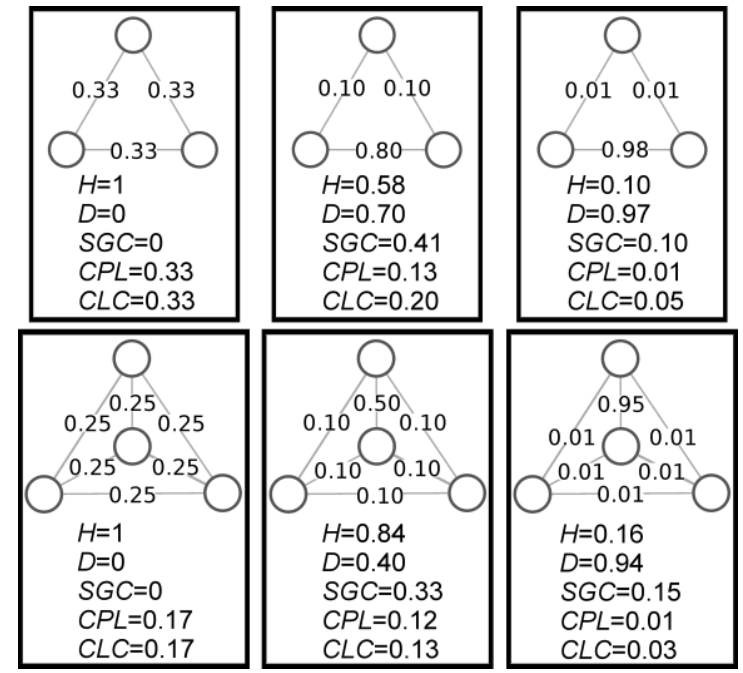

Figure 1. Figure 1. Examples of simple graphs an their values of balanced $(H)$ and unbalanced $(D)$ weigh distribution, complexity $(S G C)$, integration $(C P L)$ and segregation $(C L C)$.
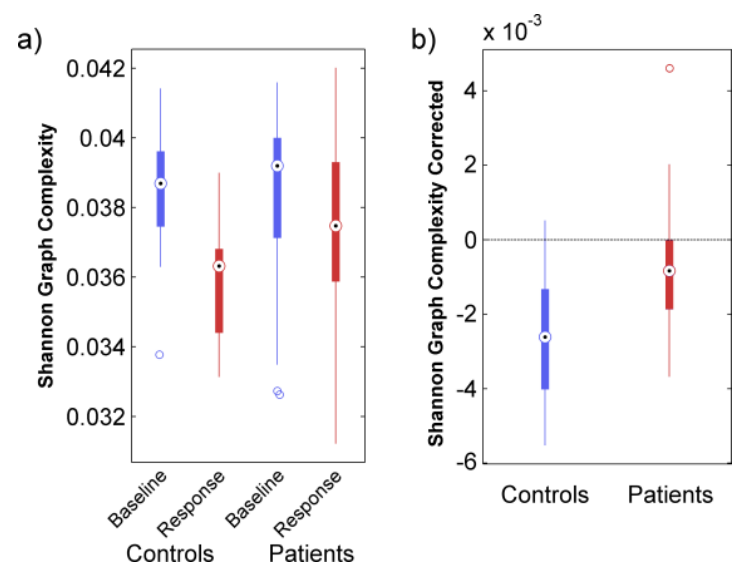

Figure 2. Figure 2. $S G C$ values in baseline and response windows for each group in theta band (a). $S G C$ change from baseline to response window for each group in theta band (b).

$0.937 \pm 0.009$ during baseline window and $0.940 \pm 0.008$ during active response. Since $H$ is higher in the response window for both groups, brain network increases the weight balance during the cognitive task ( $H=1$ for totally balanced weigh networks). This finding is related to the segregation of the network. Although most of network parameters, such as segregation, are strongly linked to network topology, it is usual that more balanced networks obtained higher segregation values (i.e. higher $C L C$ values). This issue is shown in Fig. 1. A segregated network can be associated with a high level of specialization in modules, which is in agreement with the network reorganization associated to a cognitive task [19]. Finally, the last conclusion is related with the only frequency band that showed significant differences between baseline and response (i.e. theta). As previously mentioned, this methodology is focused on the study of P3a waves (only target tones were considered). According to previous studies, P3 generation elicits important spectral changes during the response window, mainly around theta band [19]. Furthermore, low frequencies, like theta band, are related to long-range interactions [20]. Consequently, the deficit on the activation response of long-range interactions 


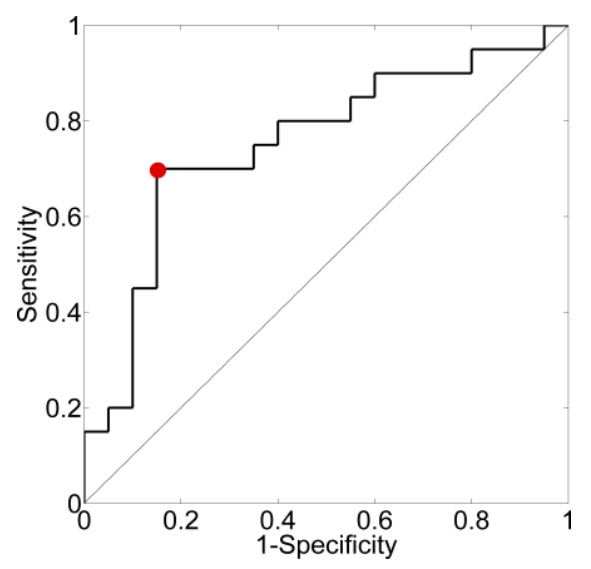

Figure 3. ROC using LOO-CV for SGC values.

TABLE I. DISCRIMINANT ABILITY FOR SGC

\begin{tabular}{|c|c|c|c|}
\hline ROC area & Sensitivity & Specificity & Accuracy \\
\hline 0.752 & 0.650 & 0.800 & 0.725 \\
\hline
\end{tabular}

might contribute to the pathological process [3]. Hence, $\mathrm{SCH}$ can be linked with an abnormal network reorganization of the neural substrates responsible of the P3a generation.

Additionally, we performed a preliminary evaluation of the $S G C$ discrimination ability in $\mathrm{SCH}$ by means of a $\mathrm{ROC}$ curve with LOO-CV (Fig. 3). The main measures related to ROC are shown in Table 1 . Sensitivity and specificity are not totally balanced (sensitivity $=0.650$, specificity $=0.800$ ), which implies lower value of false positives than false negatives, for a balanced population as in this case. In addition, $S G C$ parameter achieved an accuracy value of 0.725. There are few studies that conducted discriminant analyses for differentiating SCH and control subjects. For instance, Bachiller et al. [21] used linear discriminant analysis (LDA) applied to Shannon spectral entropy to achieve an accuracy of 0.77 . In other study, Sabeti et al. [22] used LDA and adaptive boosting with five spectral and nonlinear features to classify $\mathrm{SCH}$ group, achieving a maximum accuracy of 0.91 . Although $S G C$ reached lower accuracy values than the studies previously mentioned, it would be interesting to study whether $S G C$ is complementary to other measures and, thus, can increase the reported classification statistics.

There are some limitations that should be mentioned. Firstly, it could be appropriate to increase the sample size. Thus, the population could be divided into training and test groups to provide more robust discriminant analyses. Furthermore, it would be desirable to study other natwork parameters to explore whether they could provide complementarity results to those obtained in the current study.

\section{CONCLUSION}

This study applied a novel network measure based on Shannon entropy. SGC quantifies the distance between a graph and two extreme weight distributions: totally balanced and totally unbalanced weight distribution. The graph complexity measure proposed in our study, $S G C$, is nondependent of the network topology. Therefore, the new approach provides complementarity to traditional network features and it can show characteristic that could be hidden for them.

\section{REFERENCES}

[1] Friston K J 1998 The disconnection hypothesis Schizophr. Res. 30 $115-25$

[2] Kim J-J, Kwon J S, Park H J, Youn T, Kang D H, Kim M S, Ee D S and Lee M C 2003 Functional Disconnection Between the Prefrontal and Parietal Cortices During Working Memory Processing in Schizophrenia : A [ 15 O ] H 2 O PET Study 919-23

[3] Gomez-Pilar J, Poza J, Bachiller A, Gómez C, Molina V and Hornero R 2015 Neural Network Reorganization Analysis During an Auditory Oddball Task in Schizophrenia Using Wavelet Entropy Entropy 17 5241-56

[4] He Y, Chen Z J and Evans A C 2007 Small-World Anatomical Networks in the Human Brain Revealed by Cortical Thickness from MRI 2407-19

[5] Uhlhaas P J and Singer W 2010 Abnormal neural oscillations and synchrony in schizophrenia. Nat. Rev. Neurosci. 11 100-13

[6] Rubinov M, Knock S a., Stam C J, Micheloyannis S, Harris a. W F, Williams L M and Breakspear M 2009 Small-world properties of nonlinear brain activity in schizophrenia Hum. Brain Mapp. $30403-$ 16

[7] Tononi G, Edelman G M and Sporns O 1998 Complexity and coherency : integrating information in the brain $6613474-84$

[8] Ponten S C, Daffertshofer a., Hillebrand a. and Stam C J 2010 The relationship between structural and functional connectivity: Graph theoretical analysis of an EEG neural mass model Neuroimage $\mathbf{5 2}$ 985-94

[9] American Psychiatric Association 2013 Diagnostic and Statistical Manual of Mental Disorders, 5th Edition: DSM-5

[10] Kay S R, Opler L A and Lindenmayer J P 1989 The Positive and Negative Syndrome Scale (PANSS): Rationale and standardisation $\mathrm{Br}$. J. Psychiatry 155 59-65

[11] Bledowski C, Prvulovic D, Hoechstetter K, Scherg M, Wibral M, Goebel R and Linden D E J 2004 Localizing P300 generators in visual target and distractor processing: a combined event-related potential and functional magnetic resonance imaging study. J. Neurosci. 24 9353-60

[12] Blanco S, Garcia H, Quiroga R Q, Romanelli L and Rosso O A 1995 Stationarity of the EEG series IEEE Eng. Med. Biol. Mag. 14 395-9

[13] Bachiller A, Poza J, Gómez C, Molina V, Suazo V and Hornero R 2015 A comparative study of event-related coupling patterns during an auditory oddball task in schizophrenia J. Neural Eng. 12016007

[14] Nunez P L, Srinivasan R, Westdorp A F, Wijesinghe R S, Tucker D M, Silberstein R B and Cadusch P J 1997 EEG coherency I: Statistics, reference electrode, volume conduction, Laplacians, cortical imaging, and interpretation at multiple scales Electroencephalogr. Clin. Neurophysiol. 103 499-515

[15] Bonchev D and Buck G A 2005 Quantitative Measures of Network Complexity Quantitative Measures of Network Complexity (In Complexity in Chemistry, Biology, and Ecology. Springer US.)

[16] Martin M T, Plastino A and Rosso O A 2003 Statistical complexity and disequilibrium 311 126-32

[17] Rubinov M and Sporns O 2010 Complex network measures of brain connectivity: Uses and interpretations Neuroimage 52 1059-69

[18] Friston K 2005 Disconnection and cognitive dysmetria in schizophrenia Am. J. Psychiatry 162 429-32

[19] Polich J 2007 Updating P300: An integrative theory of P3a and P3b Clin. Neurophysiol. 118 2128-48

[20] Uhlhaas P J, Roux F, Rodriguez E, Rotarska-Jagiela A and Singer W 2010 Neural synchrony and the development of cortical networks Trends Cogn. Sci. 14 72-80

[21] Bachiller A, Díez A, Suazo V, Domínguez C, Ayuso M, Hornero R, Poza J and Molina V 2014 Decreased spectral entropy modulation in patients with schizophrenia during a P300 task. Eur. Arch. Psychiatry Clin. Neurosci. $264533-43$

[22] Sabeti M, Katebi S and Boostani R 2009 Entropy and complexity measures for EEG signal classification of schizophrenic and control participants Artif. Intell. Med. 47 263-74 